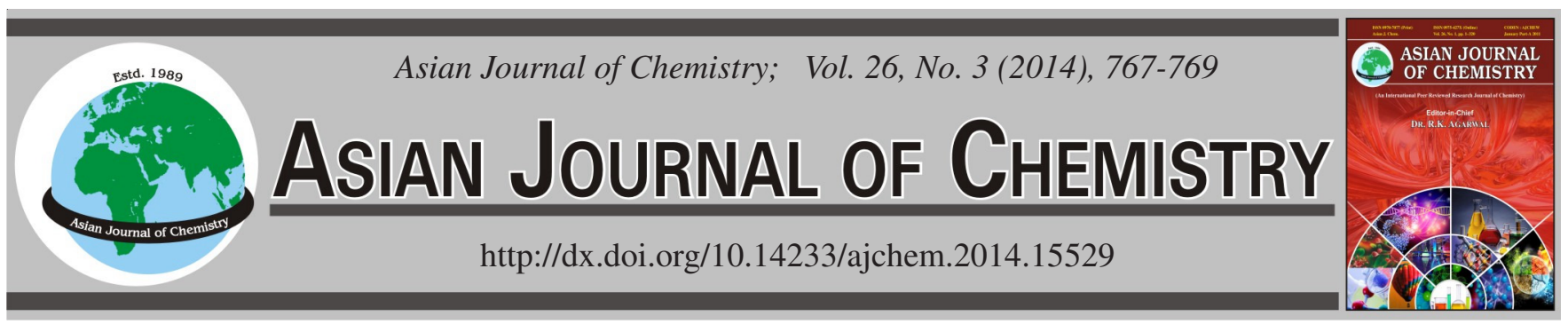

\title{
Structure and Performance Study of Polypeptide-Based Film Modified by Blending with Poly(lactic acid-co-glycolic acid)
}

\author{
Guo-Quan Zhu, Fa-Gang Wang ${ }^{*}$, Ke-Jing Xu, Qiao-Chun Gao and Yu-Ying Liu
}

School of Materials Science and Engineering, Shandong University of Technology, Zibo 255049, P.R. China

*Corresponding author: E-mail: fagangwang@126.com

Received: 23 March 2013;

Accepted: 16 July 2013;

Published online: 30 January 2014;

AJC-14633

\begin{abstract}
A series of poly $(\gamma$-benzyl L-glutamate) (PBLG)/poly(lactic acid-co-glycolic acid) (PLGA) blend films with different poly(lactic acid-coglycolic acid) mole content were prepared by casting the polymer blend solution in chloroform. Surface morphologies of PBLG/PLGA blend films were researched by scanning electron microscopy. Thermal, mechanical and chemical properties of PBLG/PLGA blend films were investigated by differential scanning calorimetry, tensile tests and surface contact angle tests. The results displayed that the introduction of poly(lactic acid-co-glycolic acid) segments could modify the structure and the performance of the polypeptide films.
\end{abstract}

Keywords: Morphology, Performance, PBLG/PLGA blend, Modified, Film.

\section{INTRODUCTION}

As known, the polymer blending has been a useful method for the improvement or modification of the physicochemical properties of polymer materials ${ }^{1-3}$. An important property of the polymer blend is the miscibility of its ingredients, as it could affect the morphology, the degradation, the permeability and the mechanical properties ${ }^{1,2}$. Lots of researches regarding the miscibility in multi-component polymer systems have already been reported. Among them, the polymer blends between biopolymers and synthetic polymers are of particular significance as they could be used as biomedical and biodegradable materials ${ }^{3-5}$.

Owing to the excellent biocompatable and biodegradable properties, polypeptides and their copolymers have received much attention for their potential applications ${ }^{6-13}$. Based on their unique structure and performance, polypeptides and their copolymers have been investigated widely in the fields of functional biomaterials, protein simulation, macromolecular conformational study, catalysis, nanoreactors and drug delivery systems $^{14-17}$. For polypeptide films, an important application is for temporary artificial skin substrates in burn therapy ${ }^{6,9}$.

The pure poly $(\gamma$-benzyl L-glutamate) (PBLG) chains are rigid and hydrophobic, while the synthetic poly(lactic acidco-glycolic acid) (PLGA) (mole ratio of lactic acid to glycolic acid is 70:30) segments are relatively flexible and hold a little hydrophilicity ${ }^{18-20}$, the introduction of PLGA segments could modify the properties of the polypeptide film and further enlarge its application fields. However, to the best of our know- ledge, no experimental work has so far been reported on the properties of PBLG/PLGA blend films. In the present work, a series of PBLG/PLGA blend films were prepared by casting the polymer blend solution in chloroform. The surface morphologies of the polymer blend films were investigated by SEM. Thermal, mechanical and chemical properties of the polymer blend films were studied by DSC, tensile tests and surface contact angle tests. It was revealed that the introduction of PLGA segments could greatly affect the properties of the polypeptide films.

\section{EXPERIMENTAL}

Poly(lactic acid-co-glycolic acid) $\left(\mathrm{M}_{\mathrm{w}}=70000\right)$, which was composed by a 70:30 ratio of lactic acid and glycolic acid units, was purchased from Jinan Daigang Biological Technology Co., Ltd. (China) and used as received. Hexane, tetrahydrofuran and 1,4-dioxane are of analytical grade and dried with sodium to remove water before use. Chloroform and other solvents are of analytical grade and used without further purification.

Synthesis of poly ( $\gamma$-benzyl L-glutamate) homopolymer: Poly ( $\gamma$-benzyl L-glutamate) homopolymer was synthesized by a standard N-carboxyl- $\gamma$-benzyl-L-glutamate anhydride (NCA) method $^{7,8}$. In brief, PBLG was obtained by the ring-opening polymerization of $\gamma$-BLG NCA initiated by triethylamine in 1,4-dioxane at room temperature for $72 \mathrm{~h}$. The reaction mixture was poured into a large volume of anhydrous ethanol. The precipitated product was dried under vacuum and then purified twice by repeated precipitation from a chloroform solution into a large volume of anhydrous methanol. The molecular 
weight of PBLG was estimated from the intrinsic viscosity measured in dichloroacetic acid ${ }^{21}$. The molecular weight of PBLG used in the study was about 80000.

Preparation of PBLG/PLGA blend film: Polymer blend films were prepared by casting a 35 wt. $\%$ polymer blend solution in chloroform onto clean glass plates and drying them under vacuum at $50{ }^{\circ} \mathrm{C}$. It is also revealed that when PLGA mole content in polymer blend is over $10 \%$, the polymer blends are not easy to form continuous film.

Measurements: SEM investigation was carried out using a scanning electron microscope (Sirin 200, FEI, Holland). Gold was sputtered on the samples in vacuum. Acceleration voltage was $10 \mathrm{kV}$ and the surface photographs of the polymer blend films were taken. DSC measurements were made on a DSC Q100 (TA, USA) differential scanning calorimeter, the temperature calibrated with indium in nitrogen atmosphere. About $7 \mathrm{mg}$ sample was weighted very accurately. The temperature was controlled within the temperature range of $30-400{ }^{\circ} \mathrm{C}$, the heating rate was $15^{\circ} \mathrm{C} / \mathrm{min}$. Tensile tests were carried out with an Instron 4468 machine (Digital Instruments Inc., USA). The crosshead speed was set to $150 \mathrm{~mm} / \mathrm{min}$. For each data point, five samples were tested and the average value was taken. The static contact angle was measured with an optical contact angle meter CAM 200 (KSV Instrument Ltd., Finland). A $5 \mu \mathrm{L}$ drop of pure distilled water was placed on the polymer blend film surface using a syringe with a 22-gauge needle. The measurements of each contact angle were performed within $10 \mathrm{~s}$ after each drop to ensure that the droplet did not soak into the compact. The surface contact angles were the mean of five determinations ${ }^{10}$

\section{RESULTS AND DISCUSSION}

Scanning electron microscopic tests: The surface morphologies of PBLG/PLGA blend films were studied by SEM technique. Fig. 1 reveals the surface morphologies of PBLG/PLGA blend films with various PLGA mole content: (a) $0 \%$, (b) $5 \%$ and (c) $10 \%$ (magnification $8000 \times$ ). As it can be seen from Fig. 1, the surface of the polymer blend films became coarser and appeared obvious phase-separation phenomenon with the introduction of the PLGA segments. As known, with the increase of the PLGA mole content in the polymer blends, on the one hand, the polypeptide chains and the PLGA segments could interact by entanglement. On the other hand, the selfaggregating action could exert between the PLGA segments. This phenomenon shows that the change of the surface morphologies of the polymer blend films could be attributed to the introduction of the PLGA segments.

Differential scanning calorimetric analysis: Table-1 displays the melting temperature of PBLG segments in the polymer blends with various PLGA mole content. As can be seen from Table-1, the melting temperature of PBLG segments in the polymer blends slightly decreased with the increase of the PLGA mole content. As mentioned above, the polypeptide chains are rigid, while the PLGA (mole ratio of lactic acid to glycolic acid is 70:30) segments are flexible, the introduction of the flexible PLGA segments with lower melting temperature could decrease the melting temperature of the PBLG segments in the polymer blends through interaction. This phenomenon indicates that the decrease of the melting temperature of PBLG segments in the polymer blends could also be attributed to the introduction of the flexible PLGA segments.

\section{TABLE-1}

MELTING TEMPERATURE OF PBLG SEGMENTS IN POLYMER BLENDS WITH VARIOUS PLGA MOLE CONTENT

PLGA content $(\mathrm{mol} \%) \quad$ Melting temperature $\left({ }^{\circ} \mathrm{C}\right)$

\begin{tabular}{cc} 
PLGA content $(\mathrm{mol} \mathrm{\%})$ & Melting temperature $\left({ }^{\circ} \mathrm{C}\right)$ \\
\hline 0 & 318.5 \\
5 & 315.8 \\
10 & 312.2 \\
\hline
\end{tabular}

Tensile tests: Table- 2 shows the tensile strength of the polymer blend films with various PLGA mole content. As is shown in Table-2, the tensile strength of the polymer blend films decreased with the increase of the PLGA mole content in polymer blends. As already noted, the rigid PBLG chains and the flexible PLGA segments could exert interaction through entanglement, suggesting that the decrease of the tensile strength of the polymer blend films was connected with the introduction of the flexible PLGA chains. Under evaluated PLGA content, the higher the PLGA content, the lower the tensile strength of the polymer blend films.

\begin{tabular}{cc} 
TABLE-2 \\
TENSILE STRENGTH OF THE POLYMER BLEND \\
FILMS WITH VARIOUS PLGA MOLE CONTENT \\
\hline PLGA content (mol \%) & Tensile strength (MPa) \\
\hline 0 & 30.2 \\
5 & 24.6 \\
10 & 20.3 \\
\hline
\end{tabular}

Surface contact angle tests: Table- 3 presents the surface contact angle of the polymer blend films with various PLGA
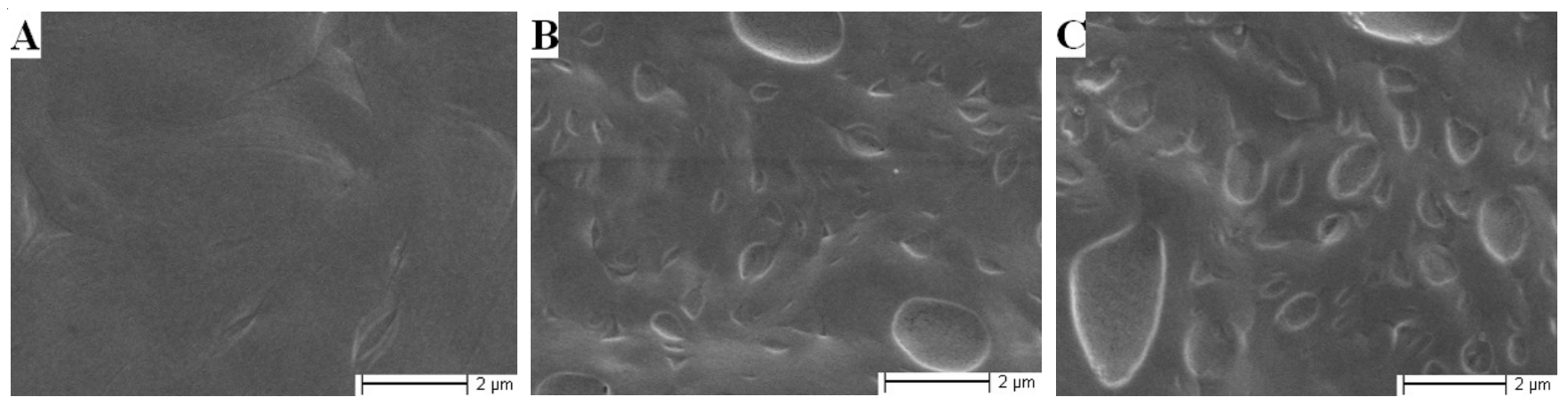

Fig. 1. SEM photographs of PBLG/PLGA blend film surface with various PLGA mole content: (a) $0 \%$, (b) $5 \%$, and (c) $10 \%$ (magnification $8000 \times$ ) 
mole content. As shown in Table-3, the surface contact angle of the polymer blend films decreased with the increase of the PLGA mole content in the polymer blends, indicating that the hydrophilicity of the polymer blend films increased. As mentioned above, the polypeptide chains are hydrophobic, while the PLGA segments hold a little hydrophilicity, the introduction of the PLGA segments could promote the hydrophilicity of the polymer blend films. This phenomenon indicates that the decrease of the surface contact angle of the polymer blend films was related with the introduction of the PLGA chains with a little hydrophilicity.

\begin{tabular}{|c|c|}
\hline \multicolumn{2}{|c|}{$\begin{array}{c}\text { TABLE-3 } \\
\text { SURFACE CONTACT ANGLE OF THE POLYMER LEND } \\
\text { FILMS WITH VARIOUS PLGA MOLE CONTENT }\end{array}$} \\
\hline PLGA content ( $\mathrm{mol} \%)$ & Surface contact angle $\left(^{\circ}\right)$ \\
\hline 0 & 79.3 \\
\hline 5 & 73.2 \\
\hline 10 & 66.5 \\
\hline
\end{tabular}

\section{Conclusion}

A series of PBLG/PLGA blend films were prepared by casting the polymer blend solution in chloroform. SEM photographs proved that the introduction of PLGA chains changed the surface morphologies of the polypeptide films. DSC tests demonstrated that the melting temperature of PBLG segments in the polymer blends decreased with the introduction of the PLGA segments. Tensile tests verified that the decrease of the tensile strength of the polymer blend films could be attributed to the introduction of the flexible PLGA segments. The surface contact angle tests attested that the introduction of the PLGA chains increased the hydrophilicity of the polypeptide films.

\section{ACKNOWLEDGEMENTS}

This work is supported by the Natural Science Foundation of Shandong Province (No. ZR2011EMM009).

\section{REFERENCES}

1. J.S. Park, J.W. Park and E. Ruckenstein, Polymer, 42, 4271 (2001).

2. Y. Nishio and R. Manley, Macromolecules, 21, 1270 (1988).

3. T. Kondo, C. Sawatari, R. Manley and D.G. Gray, Macromolecules, 27, 210 (1994).

4. C. Sawatari and T. Kondo, Macromolecules, 32, 1949 (1999).

5. K. Lio, N. Minoura and M. Nagura, Polymer, 36, 2579 (1995).

6. K. Jokei, M. Oka, T. Hayashi and Y. Miyachi, Eur. Polym. J., 35, 945 (1999).

7. D.M. Tang, J.P. Lin, S.L. Lin, S.N. Zhang, T. Chen and X.H. Tian, Macromol. Rapid Commun., 25, 1241 (2004).

8. J.P. Lin, G.Q. Zhu, X.M. Zhu, S.L. Lin, T. Nose and W.W. Ding, Polymer, 49, 1132 (2008).

9. Y. Miyachi, K. Jokei, M. Oka and T. Hayashi, Eur. Polym. J., 35, 767 (1999).

10. L.Q. Bai, L.J. Zhu, S.J. Min, L. Liu, Y.R. Cai and J.M. Yao, Appl. Surf. Sci., 254, 2988 (2008).

11. G.Q. Zhu, L. Feng and S.N. Zhang, J. Macromol. Sci. A, 46, 694 (2009).

12. G.Q. Zhu, Chem. Pap., 64, 34 (2010).

13. G.Q. Zhu, Q.C. Gao, Z.H. Li, F.G. Wang and H. Zhang, Chem. Pap., 64, 776 (2010).

14. Z.S. Gao, A. Desjardins and A. Eisenberg, Macromolecules, 25, 1300 (1992).

15. X.F. Zhong, S.K. Varshney and A. Eisenberg, Macromolecules, 25, 7160 (1992).

16. G.Q. Zhu, F.G. Wang, Y.Y. Liu and Q.C. Gao, Fiber. Polym., 11, 819 (2010).

17. M. Moffitt and A. Eisenberg, Macromolecules, 30, 4363 (1997).

18. J.S.C. Loo, C.P. Ooi and F.Y.C. Boey, Biomaterials, 26, 1359 (2005).

19. F. Ganji and M.J. Abdekhodaie, Carbohydr. Polym., 80, 740 (2010).

20. A.A. Ignatius and L.E. Claes, Biomaterials, 17, 831 (1996).

21. A. Abe and T. Yamazaki, Macromolecules, 22, 2138 (1989). 